This article has been accepted for publication in Archives of Disease in Childhood following peer review. The definitive copyedited, typeset will be available online at: https://doi.org/10.1136/archdischild-2018$\underline{315647}$

Reducing healthcare conflict: outcomes from using the Conflict Management Framework

Corresponding author: Liz Forbat, University of Stirling, Stirling, FK9 4IA, Scotland, UK.

Elizabeth.forbat1@stir.ac.uk 01786467796

Authors: Liz Forbat, University of Stirling, Stirling, UK; Sarah Barclay, Medical Mediation Foundation, London, UK.

Keywords: multidisciplinary care, conflict, ethics, health services research.

Word count: 3071 
[Type here]

\section{Reducing healthcare conflict: outcomes from using the Conflict Management Framework}

\section{Abstract}

Objective To test a new conflict management framework, to help staff identify and de-escalate conflict between staff and patients/families.

Design Before/after study reporting staff quality of life, frequency/severity of conflicts and qualitative interviews on using the Framework. Data were collected from May 2017-September 2017.

Setting A paediatric oncology department day-patient and 23 bed in-patient ward

Intervention A two-stage Conflict Management Framework used by staff at daily handovers to identify and then manage conflict cases with families.

Results Staff found the Conflict Management Framework helpful in identifying and de-escalating conflicts. The number of conflicts reported decreased by $64 \%$ from baseline to follow-up. Communication regarding conflict identification improved. Reports of staff burnout decreased between the two time-points ( $n=55$ at baseline, $n=31$ at follow-up, $p=0.001)$. Scores on compassion and secondary traumatic stress did not change.

Conclusions The Conflict Management Framework substantially reduces the incidence of conflicts and is an acceptable approach for staff. Continued use of the Framework would require it to be fully integrated into the working of the ward, which would need to include senior medical buy-in. Further refinements to the Framework have been made and will be tested in four UK sites during 2018/19 
[Type here]

\section{BACKGROUND}

Serious conflicts in paediatric services have resulted in 'intense national and international scrutiny'. ${ }^{[1 \mathrm{p} 1891]}$ Conflicts between patients, families and staff in paediatric health services are damaging to everyone involved: the child, family members and treating clinicians. Advances in life-sustaining interventions mean that more babies and children live longer, access oncology services for many years, but often live with greater morbidity. The clinical implications include an increased frequency in difficult decision-making regarding the benefits versus the burdens of intensive and invasive treatment, especially when curative treatment is no longer possible. Such circumstances have been brought into sharp focus by cases such as Ashya King ${ }^{[2]}$ where the parents and healthcare professionals disagreed about the benefits and burdens of proton beam therapy to treat his cancer. Such disagreements can and do lead to communication breakdown between clinicians, patients and relatives, as well as between clinicians themselves. The multipartner nature of much paediatric work such as the triad dynamic of clinicians, patient and relatives may increase the potential for disagreement and conflict. ${ }^{[3]}$

Paediatric conflicts tend to escalate through three distinct phases (mild, moderate, severe) if not recognised and managed early and such conflicts can have long-lasting impact. ${ }^{[4]}$ Time taken up with managing conflict can be considerable ${ }^{[5]}$ with communication breakdown, disagreements over treatment and unrealistic expectations cited as common causes.

If conflict is not identified and resolved at an early stage, differences in viewpoint can become entrenched and lead to court action or public confrontation, exemplified by recent cases internationally. ${ }^{[2-10]}$ The use of court interventions is both financially and emotionally damaging to all parties. There is little recognition or support within health service systems to manage conflict in paediatric services but there is an urgent need to identify acceptable and effective methods for doing so to support families and staff to identify and manage it. The aim of this study was to build on earlier work in training staff, to implement and test a novel approach to paediatric staff managing conflict called the Conflict Management Framework (CMF). 
[Type here]

\section{The Conflict Management Framework (CMF)}

The CMF was developed to assist clinicians manage conflicts and prevent escalation. It has two stages. Stage one focuses on prompting staff to be aware of and avoid the triggers for conflict. It is used at daily huddles/hand-overs. If conflict has been identified, the framework prompts staff to engage with the family as soon as possible, explore their concerns and agree a communication plan with them, e.g. agreeing how often they will be updated about their child's care and by whom. By recognising what triggers conflict, engaging with a family as quickly as possible and agreeing a plan of action, the aim was to prevent conflicts from escalation to Stage Two.

Stage two is a four-step process to be followed if a conflict continues to escalate and has led to communication breakdown between patient(s)/family members and the clinical team which is preventing the treating team from providing optimal care. At stage two, conflict is recognised as serious so senior hospital managers are informed of the case, and if necessary so too are child protection teams, legal services and hospital security.

Daily use of the CMF was championed by a senior nurse ensuring that the appropriate actions were taken, and that key staff at ward and management level were kept informed for Stage 2 cases.

Four days of training in conflict management, using an approach and materials with proven efficacy, ${ }^{[11]}$ were provided by the study team prior to commencing use of the CMF. The team has conducted previous work on the incidence and escalation of conflict in paediatric services in the UK. The first three days provided six-hours of training to senior clinicians and managers across the hospital, including approximately 10 staff from the paediatric oncology team. Oncology staff unable to attend these sessions were offered sessions by the ward educator using the same training materials, content and learning outcomes. Training was undertaken by approximately half the ward staff. Training focused on two key elements: (i) recognising conflict and how it escalates, (ii) empathy and perspective taking, (iii) communication skills, primarily exploring the family's point of view before explaining the clinician/hospital standpoint. The fourth day 
[Type here]

focused solely on introducing the CMF to the oncology team and training them in how to incorporate it into daily ward routine.

\section{METHODS}

This study was conducted using pre/post measures. A mixed-method approach to measurement was adopted with quantitative outcome measures and qualitative interviews. Following published conflict work, the theoretical basis for the tool and the outcome measures focus on communication and empathy as core constructs.

\section{Participants and measures}

The CMF was tested in a paediatric oncology department day-patient and 23 bed in-patient ward in the Princess Margaret Hospital, Perth, Australia.

Frequency and severity of conflicts were collected using a tool used in previous conflict work, ${ }^{\left[{ }^{[5}\right.}$ and completed on a daily basis by clinical staff. The tool recorded the number, duration, causes and severity of conflict cases, time taken to manage them and staff involved in managing the case. Training was provided to the team on several occasions to assist them in using this data collection tool.

Staff empathy and compassion was measured on the PROQOL (Professional Quality of Life Scale). This has three sub-scales compassion satisfaction and compassion fatigue, burnout and secondary traumatic stress. Consequently, the tool connects with recent concerns regarding compassion in UK healthcare, ${ }^{[1213]}$ and the approach which patients and families expect from healthcare professionals. ${ }^{[14]}$ Because each scale is psychometrically unique, they cannot be combined with the other scores. It has good psychometric properties with good validity data and reliability of with the three scales having an alpha of $.87 \mathrm{~m} .72$ and .80 respectively. All staff in the in-patient unit were invited to complete it. We hypothesised that staff quality of life would increase from baseline to follow-up. 
[Type here]

Perceptions of the CMF were gathered via interviews with ten staff, including doctors, nurses and allied health professionals. The sample was drawn purposively from the oncology ward, to capture a range of perspectives on the CMF. Staff were invited to interview by the ward's study co-ordinator. Interviews focused on: ease of use, effectiveness, impact on team management and morale, confidence in managing conflict, and whether CMF use succeeded in de-escalating or resolving conflict. Staff confidence and perceived skill in identifying conflict early and managing it as a team was also explored, alongside feedback on revisions to the CMF to increase the effectiveness and/or usability of the tool.

\section{Data collection}

Data were collected for one month at baseline (April/May 2017) and one month at follow-up (August/September 2017). Recordings of frequency and severity of conflicts were completed by ward staff. Staff perceptions of the CMF was gathered using one-off audio-recorded qualitative interviews, conducted on the oncology unit. Purposive sampling sought to recruit a selection of staff of different levels of seniority for the interviews. Questions focused on the implementation of the CMF on identifying conflicts, responding to conflict, team communication, completing the CMF paperwork and any changes that would strengthen the CMF. Interviews were conducted by a female researcher with a track-record in healthcare conflict research, and a PhD in health services research. The interviewer had liaised with some interviewees throughout the course of implementation, and had been a co-trainer on the conflict course prior to commencement. All interviewees were provided with information sheets, clearly describing the purpose of the CMF and study. Interviews lasted between 10-20 minutes; transcripts were not returned to participants for comment.

\section{Analysis}

Descriptive statistics were used to analyse and report the frequency/severity and time taken managing conflicts. The PRoQOL was analysed using Mann Whitney $U$ tests. Paired analysis was not possible due to not all staff providing identifiers to enable pre/post matching of the data.

An alpha level of $p=0.05$ was set for all statistical tests, and data were managed using SPSS version 24 . 
[Type here]

Thematic analysis was used for the transcribed interview data. Inductive analysis proceeded through a fivestage process of thematic analysis. ${ }^{[15]}$ Staff and relative data were analysed separately. Stage 1 involved familiarisation with the dataset. Stage 2 involved identifying a thematic framework. Stage 3 involved indexing the data with reference to the thematic framework. In Stage 4, responses were synthesised from across respondents into a working grid of themes. Stage 5 focused on data interpretation and finalisation of key emergent themes. Qualitative data were coded and analysed in the software package Nvivo version 10 by one researcher. Saturation of core themes was reached within the sample interviewed.

\section{Ethical considerations}

Approval for this study was granted by the Western Australia Child and Adolescent Human Research Ethics committee and R\&D department prior to study commencement (PRN: RGS00041).

Interview quotes are presented without identifiers to preserve the confidentiality of interviewees.

\section{RESULTS}

\section{Number, severity and staff time in managing conflicts}

A clinically significant decrease in conflict incidents was observed from baseline to follow-up, dropping $64 \%$ (from 22 to 8). The overall severity and time taken in managing the conflicts had increased (see Table 1 below) due to one conflict during the follow-up period, a Stage 2 case, which accounted for 990 minutes (80\%) of the time. Conflict incidence and severity are described in Table 1.

The number of families involved in conflict dropped substantially at follow-up, as did the number of days with conflicts (22 at baseline and 8 at follow-up). Dominant causes of conflict were 'family micro-managing care' and 'communication breakdown'. Staff reported for example:

\footnotetext{
"Mother expressed ongoing frustrations + concerns around lack of communication by both medical + nursing staff around care and management of [child]. Mother expressed lack of communication around IV antibiotics especially" (case note of CMF Stage 2 case)
} 
[Type here]

Of the 17 staff invited to interview, nine participated (two doctors, five nurses, one social worker and one clinical psychologist). Qualitative data indicated that the training and CMF facilitated the early identification and management of low-level conflicts, resulting in only more severe conflicts being reported. For many this involved also facilitating earlier interventions for managing conflict, recognising that it had the potential to stop conflicts escalating:

I definitely think there has been a positive impact, I think we have some patients and parents where we've had low level conflict that if we'd not addressed would have continued to escalate. (Interviewee 6)

Staff reflected that the CMF had increased their confidence in managing conflict. At times this stemmed from feeling that discussing conflict was an acceptable part of team handover, for others, it had enabled more of a focus on positively managing difficult situations:

[The CMF] has given people strategies to deal and certainly myself it's changed my approach from wanting to fix to the listening and trying to walk their walk if you like, so to look at it from their perspective. [...] It increased my personal confidence comfort with dealing with some issues (Interviewee 3)

The impact on morale was reflected upon by some interviewees:

I'd like to think that's its improved morale. (Interviewee 4)

For some staff, adopting the CMF, and being able to discuss and address conflict head-on, had led to a change in ward culture, whereby parents' concerns were explored more frequently and more gently:

I think it's a little bit of a culture shift. (Interviewee 7)

Another interviewee indicated that the framework had led to senior managers adopting new ward rounds too, indicating a cultural shift in open and ongoing communication with families to identify and address concerns early on. 
[Type here]

Table 2 outlines the staff involved in managing conflicts during baseline and follow-up data collection. At follow-up, nurses' involvement reduced substantially, and multidisciplinary involvement increased. This indicates that communication and management of conflicts has begun to be acknowledged as a team concern.

\section{Staff compassion and empathy}

Outcomes for staff work-related quality-of-life were analysed. As Table 3 below demonstrates there was a statistically significant decrease in burnout at follow-up $(p<0.001)$. Scores in compassion and traumatic stress improved, but not at a statistically significant level. Lower scores indicate lower distress.

\section{Continued use of the CMF}

Interviewees were asked their thoughts on whether the CMF would be used after the end of the study. Many felt that the approach was very helpful and expressed a desire to continue using it:

I think it would be helpful to carry on using it, and because I think this is the first time that I can see that the team has been given a tool to work with these challenging situations and what I personally like about it [...] is that it's a reflective tool, is that the act of identifying it makes one think about it. (interviewee 4)

Several staff felt that the paperwork was overly complex:

I felt like there was lots of wording rather than it being kind of bullet point [...] It felt like there was lots of documentation going on about exactly the same thing but in different parts. (Interviewee 8)

Some suggested that documenting only more substantial conflicts would be helpful, and more minor incidents could be verbally reported but not require the completion of the CMF Phase One paperwork. This modification by staff fitted with the intended use of the CMF when conflicts were escalating. This adaptation had already begun to be taken on by the team, with lengthy handovers about conflict being truncated: 
[Type here]

Initially we were having lots of conflict information handed over in our team handover and it was making the handover last for too long. Everybody was getting off shift late, and that makes people negative towards what's going on so we've kind of gone very low level in handover so we'll say there is conflict. [...] If there's conflict it would be handed over at the bedside and then during bedside handover as the nurses have been on the previous shift you then get all the details and then obviously we have access to the file to then go over to look at the history. (Interviewee 6)

Several interviewees felt that the CMF could only work if senior clinicians and hospital managers were committed to the process. Involvement of the medical team and senior management had been very well received:

I think there's enough awareness and I think there's enough people in leadership positions that have been driving it that it becomes more ingrained in our day to day work. (interviewee 3)

\section{DISCUSSION}

The Conflict Management Framework substantially reduced the number of conflicts over the course of six months, and a statistically significant change in staff burn-out was observed. Compassion satisfaction and secondary traumatic stress did not change at a statistically significant level.

The CMF addresses calls for early intervention in managing conflict ${ }^{[1]}$ and could act as a tool to obviate financially and emotionally costly court action, ${ }^{[16]}$ or complexity of arbitration.${ }^{[1718]}$ Burnout is a recognised potential sequelae to work-related stress, with high prevalence in specialities such as intensive care. ${ }^{[19]}$ Staff burnout is associated with reduced patient safety, ${ }^{[20]}$ making it an important focus for interventions. Further, the CMF has the potential to reduce the substantial burdens of staff time/cost associated with managing conflict ${ }^{[5]}$ and emotional burden on staff and families. ${ }^{[21-23]}$ Communication breakdown is a 
[Type here]

recognised component in conflict escalation, ${ }^{[424]}$ and was evident in the data from this study. Training in both communication ${ }^{[25]}$ and conflict management ${ }^{[26]}$ need to be viewed as core skills in paediatric specialities where there is complex case-management of critically ill children. Staff reported that their increased skill in communicating with families was a marked advantage having taken part in the training. Notably listening and perspective-taking were both cited as important, reflecting what Ranjan et al refer to as frequently neglected communication skills. ${ }^{[27]}$ This study has successfully extended previous work where training was offered as a stand-alone intervention ${ }^{[11]}$ by adding the CMF as a framework that staff can adopt when they identify conflict.

Benchmarking this site to others regarding number of conflicts observed is impossible, since there is little available data on conflict incidence where service size and patient throughput are reported. Where data does exist, high levels of conflict are linked with poorer patient prognosis and the need for complex multidisciplinary care ${ }^{[5]}$ Thus, while it is not possible to speculate whether incidence of 22 or eight might be considered on a par with other services, the reduction was substantial, but the remaining conflicts warrant ongoing intervention and concern. Further prevalence studies are required to provide helpful benchmarking within and across services to drive up standards and promote proactive conflict management strategies.

Although interviewees roundly praised the training they had received prior to the CMF being implemented, the data may be bias as the interviewer had co-facilitated the training sessions. The study's generalisability is limited by receiving insufficient outcome measures to enable fully powered analysis. A power calculation was not used to inform sample size for the collation of baseline or follow-up incidence and severity of conflict.

Further work is required to understand family perspectives on the use of the CMF, and refinements which would improve their experiences when disagreements occur. A full economic evaluation of the CMF should also be conducted whereby the costs of staff training (including back-fill for those in clinical roles) is assessed against the savings in time spent managing conflict, legal fees, and expenses such as staff sickness. 
[Type here]

Although there is some evidence regarding the incidence of conflict in different clinical specialities, ${ }^{[5]}$ further work could helpfully examine the culture of different departments and hospitals to consolidate learning on the contextual features which influence when conflict breeds or is stifled. Consequently, a multi-site and cross-speciality study of the CMF is warranted, to understand its implementation potential.

\section{CONCLUSION}

The principles of the CMF of early intervention and de-escalation are core drivers in reducing the negative sequalae of conflict. Since many of the conflicts were focused around communication, it would be prudent for hospitals to ensure ongoing training in staff/family communication. For continued use of the Framework, senior leadership is required at the ward and hospital level, including buy-in from medical colleagues. The implementation benefited from such leadership during this study. Further development and refinement of the CMF is being conducted in four UK paediatric sites in 2018/9 to improve its usability and impact. Following a number of recent high profile court cases involving disputes between clinicians and parents, the need to adopt more structured approaches to identifying and managing conflict has been expressed by clinicians, medical ethicists and judges. ${ }^{[1617]}$ Structured approaches to identifying principle concerns and subsequent actions ${ }^{[28]}$ could incorporate the CMF as an effective approach to managing conflict in these complex cases. 
[Type here]

What is already known on this topic?

- Conflict is a frequent component of paediatric services and can have severe consequences for staff, patients, families and services

- Few strategies exist to enable clinicians to manage conflict

- If conflict is not managed effectively, cases escalate and can result in high profile court action

\section{What this study adds}

- Incidence and severity of conflict can be reduced in paediatric oncology by using the Conflict Management Framework

- Staff find the Conflict Management Framework helpful

- Using the Conflict Management Framework reduces staff burnout 


\section{REFERENCES}

1. Wilkinson D, Savulescu J. Alfie Evans and Charlie Gard-should the law change? BMJ (Clinical research ed) 2018;361:k1891. doi: 10.1136/bmj.k1891 [published Online First: 2018/05/03]

2. O'Brien A, Sokol DK. Lessons from the Ashya King case. British Medical Journal 2014;349:g5563. doi: 10.1136/bmj.g5563 [published Online First: 2014/09/12]

3. Greene M, Adelman R. Beyond the Dyad: Communication in Triadic (and more) Medical Encounters. In: DiMatteo ML, ed. The Oxford Handbook of Health Communication, Behavior Change, and Treatment Adherence Oxford: Oxford Handbooks, 2013:136-54.

4. Forbat L, Teuten B, Barclay S. Conflict escalation in paediatric services: Findings from a qualitative study. Arch Dis Child 2015;100(8):769-73. doi: 10.1136/archdischild-2014-307780

5. Forbat L, Sayer C, McNamee $P$, et al. Conflict in a paediatric hospital: a prospective mixed-method study. Arch Dis Child 2016;101(1):23-7. doi: 10.1136/archdischild-2015-308814 [published Online First: 2015/11/11]

6. McDougall R, Delany C, Gillam L. When doctors and parents disagree: Ethics, paediatrics and the zone of parental discretion. London: Routledge 2016.

7. Verhagen AA, de Vos M, Dorscheidt JH, et al. Conflicts about end-of-life decisions in NICUs in the Netherlands. Pediatrics 2009;124(1):e112-9. doi: 10.1542/peds.2008-1839 [published Online First: 2009/07/01]

8. Ruppe MD, Feudtner $C$, Hexem KR, et al. Family factors affect clinician attitudes in pediatric end-of-life decision making: a randomized vignette study. Journal of pain and symptom management 2013;45(5):832-40. doi: 10.1016/j.jpainsymman.2012.05.005 [published Online First: 2012/09/29]

9. Perkin RM, Young T, Freier MC, et al. Stress and distress in pediatric nurses: lessons from Baby K. American journal of critical care : an official publication, American Association of Critical-Care Nurses 1997;6(3):225-32. [published Online First: 1997/05/01]

10. Spotova A, Husakova K, Hraskova A, et al. Treatment Refusal in Pediatric Oncology. Klinicka onkologie : casopis Ceske a Slovenske onkologicke spolecnosti 2017;30(5):380-85. doi: 10.14735/amko2017380 [published Online First: 2017/10/17]

11. Forbat L, Simmons J, Sayer C, et al. Training paediatric health care staff in recognising, understanding and managing conflict with patients and families: Findings from a survey on immediate and sixmonth impact. Arch Dis Child 2016;102(3):250-54.

12. Francis R. Report of the Mid Staffordshire NHS Foundation Trust Public Inquiry. London: The Stationery Office, 2013.

13. Francis R. Independent Inquiry into Care Provided by Mid Staffordshire NHS Foundation Trust January 2005-March 2009. London: The Stationery Office, 2010.

14. Griffiths J, Speed S, Horne M, et al. 'A caring professional attitude': what services users and carers seek in graduate nurses and the challenge for educators. Nurse Education Today 2012;32(2):121-27.

15. Braun V, Clarke V. Using thematic analysis in psychology. Qualitative Research in Psychology 2006;3:77101.

16. Wallis C. When paediatricians and families can't agree. Arch Dis Child 2018;103(5):413. doi: 10.1136/archdischild-2017-314414

17. Wheeler R. Response to 'When paediatricians and families can't agree'. Arch Dis Child 2018;103(5):410. doi: 10.1136/archdischild-2017-314612

18. Lajeunesse M. Prevention of conflict is better when there is no cure. Arch Dis Child 2018;103(5):415.

19. Embriaco N, Papazian L, Kentish-Barnes N, et al. Burnout syndrome among critical care healthcare workers. Current opinion in critical care 2007;13(5):482-8. doi: 10.1097/MCC.0b013e3282efd28a [published Online First: 2007/09/01] 
[Type here]

20. Hall LH, Johnson J, Watt I, et al. Healthcare Staff Wellbeing, Burnout, and Patient Safety: A Systematic Review. PLOS One 2016:1-12. doi: doi.org/10.1371/journal.pone.0159015 [published Online First: 8th July 2016]

21. McDougall R, Notini L, Phillips J. Conflicts Between Parents and Health Professionals About a Child's Medical Treatment: Using Clinical Ethics Records to Find Gaps in the Bioethics Literature. Journal of bioethical inquiry 2015;12(3):429-36. doi: 10.1007/s11673-015-9643-7 [published Online First: 2015/07/03]

22. Brinkert R. A literature review of conflict communication causes, costs, benefits and interventions in nursing. Journal of Nursing Management 2010;18(2):145-56. doi: 10.1111/j.13652834.2010.01061.x

23. Almost J, Wolff AC, Stewart-Pyne A, et al. Managing and mitigating conflict in healthcare teams: an integrative review. J Adv Nurs 2016;72(7):1490-505. doi: 10.1111/jan.12903 [published Online First: 2016/01/30]

24. Studdert DM, Mello MM, Burns JP, et al. Conflict in the care of patients with prolonged stay in the ICU: types, sources, and predictors. Intensive care medicine 2003;29(9):1489-97. doi: 10.1007/s00134003-1853-5 [published Online First: 2003/07/25]

25. Fallowfield L, Jenkins V. Communicating sad, bad, and difficult news in medicine. Lancet (London, England) 2004;363(9405):312-9. doi: 10.1016/s0140-6736(03)15392-5 [published Online First: 2004/01/31]

26. Saltman DC, O'Dea NA, Kidd MR. Conflict management: a primer for doctors in training. Postgrad Medical Journal 2006;82(963):9-12. doi: 10.1136/pgmj.2005.034306 [published Online First: 2006/01/07]

27. Ranjan P, Kumari A, Chakrawarty A. How can Doctors Improve their Communication Skills? Journal of Clinical and Diagnostic Research : JCDR 2015;9(3):JE01-JE04. doi: 10.7860/JCDR/2015/12072.5712

28. Wilkinson D, Barclay S, Savulescu J. Disagreement, mediation, arbitration: resolving disputes about medical treatment. The Lancet 2018;391:2302-05. 
[Type here]

Table 1: Conflict incidence, severity and time

\begin{tabular}{|l|l|l|}
\hline & \multicolumn{1}{|c|}{ Pre $\mathbf{( n = 2 2 )}$} & \multicolumn{1}{|c|}{ Post $(\mathbf{n}=\mathbf{8})$} \\
\hline Severity & Median: 3 & Median: 5 \\
& Range: $2-5.5$ & Range: $2-6$ \\
\hline Time (mins) & Total: 835 & Total: 1240 \\
& Range: $5-150$ & Range: $10-180$ \\
\hline
\end{tabular}

Table 2: Staff involved in managing conflicts

\begin{tabular}{|l|l|}
\hline \multicolumn{2}{|c|}{ Staff group } \\
\hline \multicolumn{1}{|c|}{ Pre (n=37) } & \multicolumn{1}{c|}{ Post (n=29) } \\
\hline Staff nurse/RN: 20 & Consultant: 7 \\
Consultant: 8 & Clinical Nurse Specialist: 5 \\
Other doctor: 5 & Manager: 3 \\
After-hours manager: 1 & Registered Nurse: 4 \\
Clinical Nurse Specialist: 1 & Social work: 2 \\
Sister: 1 & Other: 1 \\
Student nurse: 1 & Chaplain: 1 \\
& Inpatient nursing director: 1 \\
& Occupational Therapist: 1 \\
& Physiotherapist: 1 \\
& Shift co-ordinator: 1 \\
& Security: 1 \\
& Refugee consultant: 1 \\
\hline
\end{tabular}

Table 3: Staff compassion, burnout and secondary traumatic stress

\begin{tabular}{|c|c|c|}
\hline \multicolumn{2}{|l|}{ ProQOL } & \multirow{2}{*}{$\begin{array}{l}\text { Statistical } \\
\text { significance }\end{array}$} \\
\hline Pre & Post & \\
\hline $\begin{array}{l}\text { Compassion satisfaction } \\
\text { ( } n=53) \\
\text { median: } 40 \text { (average) } \\
\text { range: } 29-48\end{array}$ & $\begin{array}{l}\text { Compassion satisfaction } \\
\text { ( } \mathbf{n}=\mathbf{3 1}) \\
\text { median: } 40 \text { (average) } \\
\text { range: } 31-50\end{array}$ & 0.863 \\
\hline $\begin{array}{l}\text { Burnout }(n=55) \\
\text { median: } 27 \text { (average) } \\
\text { range: } 15-39\end{array}$ & $\begin{array}{l}\text { Burnout }(\mathbf{n}=\mathbf{3 1}) \\
\text { median: } 22 \text { (low) } \\
\text { range: } 14-35\end{array}$ & 0.001 \\
\hline $\begin{array}{l}\text { Secondary traumatic } \\
\text { stress ( } \mathbf{n}=\mathbf{5 3}) \\
\text { median: } 21 \text { (low) } \\
\text { range: } 15-31\end{array}$ & $\begin{array}{l}\text { Secondary traumatic stress } \\
\text { ( } \mathrm{n}=\mathbf{3 1} \text { ) } \\
\text { median: } 21 \text { (low) } \\
\text { range: } 14-36\end{array}$ & 0.214 \\
\hline
\end{tabular}

